\title{
In-situ Neutron Reflectometry Study on Adsorption of Glucose Oxidase at Mesoporous Aluminum Oxide Film
}

\author{
Akira Yamaguchi, ${ }^{* \dagger}$ Kazuya Katayama,* and Stephen A. HolT** \\ *Institute of Quantum Beam Science, Ibaraki University, 2-1-1 Bunkyo, Mito, Ibaraki 310-8512, Japan \\ **Australian Centre for Neutron Scattering, Australian Nuclear Science and Technology Organisation (ANSTO), \\ Lucas Heights, New South Wales 2234, Australia
}

\begin{abstract}
In the present study, the adsorption of glucose oxidase (GOD) to a mesoporous aluminum oxide (MAO) film was examined with in-situ neutron reflectometry (NR) measurements. The MAO film was deposited on a cover glass slip and a Si disc, and its pore structure was characterized by X-ray reflectometry (XRR) and NR. The Si disc with MAO film was applied for an in-situ NR experiment, and its NR profiles before/after adsorption of GOD were continuously measured with a flow cell. The results indicated that the negatively-charged GOD molecules hardly penetrate into the narrow pore channel (pore diameter $=c a .10 \mathrm{~nm}$ ) with opposite surface charge.
\end{abstract}

Keywords Mesoporous aluminum oxide, myoglobin, adsorption, neutron reflectometry

(Received May 11, 2020; Accepted June 9, 2020; Advance Publication Released Online by J-STAGE June 12, 2020)

\section{Introduction}

Protein adsorption at an inorganic nanoporous material is a fundamental process in the chromatographic separation of proteins and synthesis of an artificial biocatalytic system. ${ }^{1-3}$ The nanoporous material possesses both an outer surface and internal pore surface, and both can be regarded as adsorption sites for protein molecules. The distribution of protein molecules at both adsorption sites affects the chromatographic separation and performance of the biocatalytic system. The protein distribution on and in nanoporous materials is thus an important subject to be clarified, and in-situ spectroscopic techniques for protein adsorption have been developed. ${ }^{4-17}$ The distribution of adsorbate on a single nanoporous particle has been often observed by microspectroscopy techniques. ${ }^{4,5}$ For a nanoporous film on a solid substrate, spectroscopic methods utilizing optical interference ${ }^{6-14}$ and waveguide mode ${ }^{15-17}$ are available for the in-situ observation of the molecular adsorption.

In-situ neutron reflectometry (NR) is one useful technique for structural characterization of an adsorption layer at a solid/ liquid interface. ${ }^{11-14}$ The scattering length densities (SLD) of $\mathrm{D}_{2} \mathrm{O}$ and $\mathrm{H}_{2} \mathrm{O}$ are quite different (Table 1), and SLD contrast between materials within the nanoporous film and solvent can be easily tuned by adjusting solvent composition of a $\mathrm{D}_{2} \mathrm{O} / \mathrm{H}_{2} \mathrm{O}$ mixture. When NR measurement is performed under a series of $\mathrm{D}_{2} \mathrm{O} / \mathrm{H}_{2} \mathrm{O}$ mixtures, simultaneous analysis of reflectivity data obtained for the $\mathrm{D}_{2} \mathrm{O} / \mathrm{H}_{2} \mathrm{O}$ mixtures can reduce ambiguities while modeling, and facilitates determination of the structural properties of the adsorption layer. ${ }^{18}$ The in-situ NR has been applied to characterize lipid membranes assembled at a surfactant-templated mesoporous silica film ${ }^{12,13}$ and adsorption of

† To whom correspondence should be addressed.

E-mail: akira.yamaguchi.sci@vc.ibaraki.ac.jp polyelectrolyte at an anodized nanoporous aluminum oxide film. ${ }^{14}$ The purpose of this study is application of in-situ NR to clarify whether negatively-charged glucose oxidase (GOD) can penetrate into pores in mesoporous aluminum oxide (MAO) film or not. The MAO film used in this study was prepared by a surfactant-templated method, ${ }^{19}$ and contained ordered pores with diameter of $c a .10 \mathrm{~nm}$. This pore size is much smaller than that of a conventional nanoporous aluminum oxide film prepared by anodization $(\geq 20 \mathrm{~nm}) .{ }^{15,20}$ We previously reported penetration of negatively-charged bovine serum albumin (BSA, $67 \mathrm{kDa}$, $14 \times 4 \times 4 \mathrm{~nm})^{21}$ into large pores (diameter: $33 \mathrm{~nm}$ ) of anodic aluminum oxide film. Since the pore diameter of the MAO film is close to molecular dimensions of GOD $(160 \mathrm{kDa}, 6 \times 5.2 \times$ $7.7 \mathrm{~nm}),{ }^{22}$ it is expected that the size-matching between GOD and pore affects the adsorption behavior of GOD.

In the present study, we deposited the MAO film on a conventional cover glass slip for structural characterization of the MAO film by X-ray reflectometry (XRR), NR, and scanning electron microscopy (SEM). For the in-situ NR experiment, we deposited the MAO film on a Si disc. The structure of the MAO

Table 1 SLD values used for the analysis of XRR and NR data

\begin{tabular}{lcc}
\hline & Neutron $/ 10^{-6} \AA^{-2}$ & X-ray $/ 10^{-6} \AA^{-2}$ \\
\hline $\mathrm{D}_{2} \mathrm{O}$ & 6.34 & - \\
$\mathrm{H}_{2} \mathrm{O}$ & -0.56 & - \\
$\mathrm{Si}$ & 2.07 & 20.7 \\
$\mathrm{SiO}_{2}$ & 3.47 & 18.9 \\
$\mathrm{Al}_{2} \mathrm{O}_{3}$ & 5.67 & 33.3 \\
$\mathrm{Glass}^{\mathrm{a}}$ & 3.6 & 21 \\
$\mathrm{GOD}$ & $3.5^{\mathrm{b}}$ & \\
\hline
\end{tabular}

a. Cover glass slip.

b. Ref. 27. 
Table 2 Structural parameters obtained from fitting the NR and XRR profiles for MAO films on glass substrates

\begin{tabular}{|c|c|c|c|c|c|c|c|}
\hline & & \multicolumn{2}{|c|}{ Al03 } & \multicolumn{2}{|c|}{ Al05 } & \multicolumn{2}{|c|}{ Al07 } \\
\hline & & NR & XRR & NR & XRR & NR & XRR \\
\hline \multirow[t]{3}{*}{ Top } & $t^{\mathrm{a}}$ & $63 \pm 3$ & $76 \pm 1$ & $30 \pm 8$ & $33 \pm 2$ & $39 \pm 1$ & $30 \pm 1$ \\
\hline & $\mathrm{SLD}^{\mathrm{b}}$ & $0.32 \pm 0.03$ & $2.3 \pm 0.1$ & $1.8 \pm 0.4$ & $8.3 \pm 0.3$ & $2.6 \pm 0.1$ & $17 \pm 1$ \\
\hline & $\sigma^{\mathrm{c}}$ & $11 \pm 6$ & $17 \pm 1$ & $55 \pm 2$ & $24 \pm 1$ & $10 \pm 1$ & $6.5 \pm 0.1$ \\
\hline \multirow[t]{3}{*}{ Main } & $t$ & $594 \pm 2$ & $646 \pm 2$ & $854 \pm 3$ & $827 \pm 1$ & $877 \pm 3$ & $893 \pm 6$ \\
\hline & SLD & $0.95 \pm 0.02$ & $2.8 \pm 0.1$ & $1.2 \pm 0.1$ & $5.8 \pm 0.1$ & $3.3 \pm 0.1$ & $21 \pm 1$ \\
\hline & $\sigma$ & $26 \pm 2$ & $23 \pm 1$ & $42 \pm 4$ & $18 \pm 1$ & $15 \pm 1$ & $13 \pm 1$ \\
\hline \multirow[t]{3}{*}{ Bottom } & $t$ & $20 \pm 3$ & $15 \pm 10$ & $58 \pm 8$ & $61 \pm 1$ & $25 \pm 40$ & $23 \pm 20$ \\
\hline & SLD & $2.5 \pm 0.2$ & $19 \pm 5$ & $3.8 \pm 0.3$ & $22 \pm 1$ & $3.6 \pm 0.2$ & $21 \pm 1$ \\
\hline & $\sigma$ & $7.7 \pm 2.8$ & $9.9 \pm 0.7$ & $16 \pm 5$ & $9.8 \pm 0.1$ & $10 \pm 16$ & $14 \pm 1$ \\
\hline \multirow[t]{2}{*}{ Total } & $t_{\mathrm{tot}}$ & $677 \pm 5$ & $737 \pm 10$ & $942 \pm 12$ & $920 \pm 2$ & $940 \pm 40$ & $946 \pm 21$ \\
\hline & $<P_{\mathrm{por}}>^{\mathrm{d}}$ & 0.83 & 0.91 & 0.76 & 0.79 & 0.41 & 0.38 \\
\hline
\end{tabular}

a. Thickness $(\AA)$. b. Scattering length density $\left(10^{-6} \AA^{-2}\right)$. c. Surface roughness $(\AA)$. d. Average porosity calculated by Eq. (2).

film on the Si disc was characterized by XRR prior to the in-situ NR experiment. In-situ NR experiments were performed in $\mathrm{D}_{2} \mathrm{O} / \mathrm{H}_{2} \mathrm{O}$ mixtures to characterize the amount and distribution of GOD adsorbed at the MAO film.

\section{Experimental}

\section{Materials and chemicals}

A square cover glass slip $(24 \times 24 \times 0.1 \mathrm{~mm})$ was purchased from Matsunami Glass Ind., Ltd., Osaka, Japan. Pluronic ${ }^{\circledR}$ F127 $\left(\begin{array}{llll}\mathrm{PEO}_{106} & \mathrm{PPO}_{70} & \mathrm{PEO}_{106}\end{array}\right)$ and glucose oxidase (GOD) were purchased from Sigma-Aldrich Japan (Tokyo, Japan). Aluminum nitrate was purchased from Fujifilm Wako Pure Chemical Corp. (Osaka, Japan). Deionized water was used for the preparation of the MAO film and for in-situ NR measurement. The polished silicon disc (100 mm in diameter and $10 \mathrm{~mm}$ in height) and $\mathrm{D}_{2} \mathrm{O}$ used for the in-situ NR measurement were supplied from Australian Nuclear Science and Technology Organisation (ANSTO, Sydney, Australia).

The details of the preparation procedures of the MAO film are described in a previous report. ${ }^{19}$ In brief, Pluronic ${ }^{\circledR}$ F127 (0.1 g) was dissolved in $2 \mathrm{~mL}$ of water containing aluminum nitrate (0.3 - $0.7 \mathrm{M})$, and the mixture was stirred at room temperature for $4 \mathrm{~h}$. This precursor solution was spin-coated on the clean glass substrate at $600 \mathrm{rpm}$ for $20 \mathrm{~s}$, and accelerated to $4000 \mathrm{rpm}$ for $60 \mathrm{~s}$. The glass substrate was dried overnight at room temperature and then calcined at $500^{\circ} \mathrm{C}$ for $6 \mathrm{~h}$ in ambient atmosphere. Hereinafter, we designated the glass substrate with MAO film as Al $x x$, where $x x$ indicates the concentration of aluminum nitrate (Table 2). For the deposition of MAO film on the $\mathrm{Si}$ disc, the spin-coating was performed at $600 \mathrm{rpm}$ for $20 \mathrm{~s}$, then accelerated to $3500 \mathrm{rpm}$ for $60 \mathrm{~s}$. The concentration of aluminum nitrate in the precursor solution was $0.7 \mathrm{M}$. The rotation speed was reduced due to heaviness of the thick and large Si disc. The calcination of the MAO film on the Si disc was performed at $350^{\circ} \mathrm{C}$ to avoid formation of a thick and inhomogeneous $\mathrm{SiO}_{2}$ layer by the thermal oxidation of the $\mathrm{Si}$ surface. The formation of ordered pores in the MAO film calcined at $350^{\circ} \mathrm{C}$ was confirmed by SEM observation. We designated the Si disc with the MAO film as Al07/Si (Table 2).

\section{Measurements}

The MAO film deposited on the cover glass slip was characterized by XRR, NR, and SEM measurements. The SEM measurements were performed with field-emission scanning

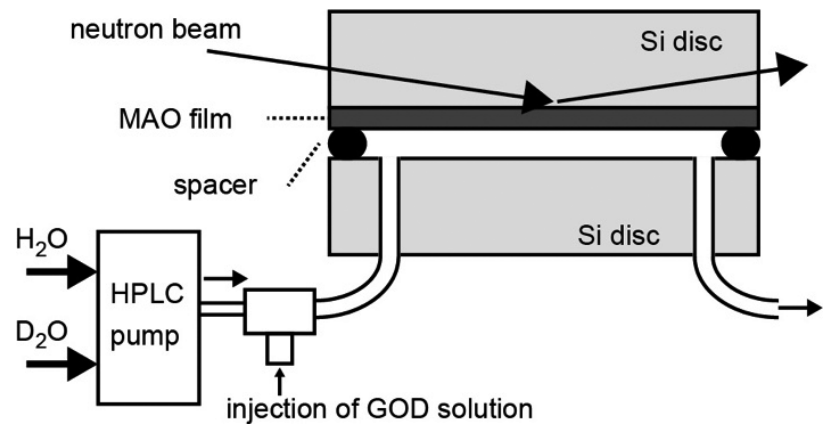

Fig. 1 Schematic illustration of solid-liquid cell used for in-situ NR measurement.

electron microscopy (FE-SEM, Hitachi S-4800). The XRR profiles were measured in air on a PANalytical X'pert Pro reflectometer at ANSTO. The XRR measurement was performed with an accelerating voltage of $45 \mathrm{kV}$, current of $40 \mathrm{~mA}$ and with $\mathrm{Cu} K \alpha$ radiation $(1.54 \AA$ ). $\quad$ NR measurements were performed at the PLATYPUS time-of-flight reflectometer at the $20 \mathrm{MW}$ OPAL reactor of the ANSTO nuclear facility ${ }^{23}$ with an incident cold neutron wavelength range from 2 to $20 \AA$. The NR measurements were performed at two different angles of incidence; 0.7 and $2.5^{\circ}$ in air conditions, and 0.9 and $4.0^{\circ}$ in solution conditions. The scattering vectors for XRR and NR were calculated by $Q=(4 \pi / \lambda) \sin \theta$, where $\lambda$ is the wavelength and $\theta$ is the angle of incidence.

The XRR and NR experiments in air were performed by irradiating an X-ray or neutron beam from the upper side of the MAO film. For the in-situ NR experiment, the Si disc with the MAO film was fitted in a solid-liquid cell (Fig. 1). ${ }^{21}$ After introduction of a solution into the cell, the NR measurement was performed at room temperature $\left(c a .25^{\circ} \mathrm{C}\right)$. The neutron beam impinged onto the MAO film by penetrating through the silicon disc. By changing the solution in the cell, NR profiles in $\mathrm{D}_{2} \mathrm{O}, 90 \% \mathrm{D}_{2} \mathrm{O}\left(\mathrm{D}_{2} \mathrm{O} / \mathrm{H}_{2} \mathrm{O} 90: 10\right.$, v/v), and $\mathrm{H}_{2} \mathrm{O}$ were recorded. Then, we introduced $10 \mathrm{wt} \%$ GOD solution $\left(90 \% \mathrm{D}_{2} \mathrm{O}\right)$ and incubated for $1 \mathrm{~h}$. After incubation, NR measurement was performed in the GOD solution. We rinsed the sample surface by successive flow of $\mathrm{D}_{2} \mathrm{O}$ into the measurement cell. Finally, NR profiles after the rinsing were recorded in $\mathrm{D}_{2} \mathrm{O}$ and $90 \%$ $\mathrm{D}_{2} \mathrm{O}$. Data reduction and modelling of the NR data were conducted using the IgorPro based macro MOTOFIT. ${ }^{24}$ The $Q$ resolutions for the analysis of XRR and NR data were fixed at 

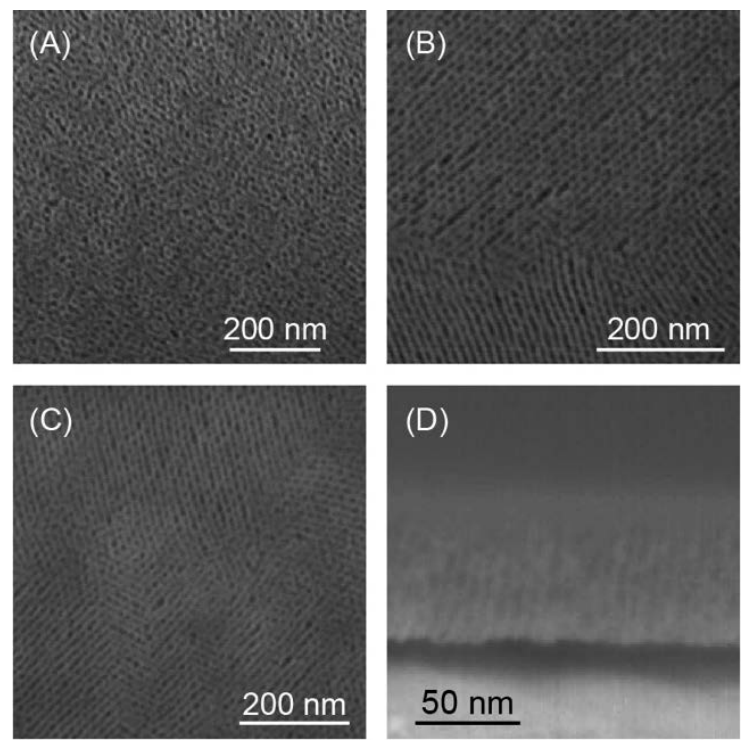

Fig. 2 SEM top views of (A) Al03, (B) A105, and (C) Al07. (D) cross-sectional SEM view of Al07.

1 and $8 \%$, respectively. SLD values used for the analysis are summarized in Table 1.

\section{Results and Discussion}

$X R R$ and NR experiments for MAO film on glass substrate

Figures 2(A) to 2(C) shows typical SEM top views of the MAO films on cover glass slips. The ordered pore structures are recognized for A105 and Al07, while Al03 shows a less ordered pore structure. The XRR and NR data recorded in air with glass substrates are shown in Fig. 3. Al03 and Al05 exhibited clear Kiessig fringes from the MAO film, while the Kiessig fringes were not seen for Al07. According to the XRR study on a mesoporous silica film prepared by a surfactant-templated method, ${ }^{25}$ all reflectivity data were analyzed by a three-layer (top, main, and bottom layers) model as schematically shown in inset of Fig. 3. As listed in Table 2, large SLD contrasts between the MAO film and glass substrate enable the observation of Kiessig fringes, due to the total film thickness, for Al03 and A105. On the other hand, the lower SLD contrasts for Al07 results in disappearance of Kiessig fringes. Herein, porosity, $P_{\text {por }}$, of each MAO layer is defined as

$$
P_{\mathrm{por}, \mathrm{i}}=\rho_{\mathrm{i}} / \rho_{\mathrm{AO}}
$$

where $\rho_{\mathrm{i}}$ is SLD of $i$-th MAO layer, and $\rho_{\mathrm{AO}}$ is SLD of pure aluminum oxide (Table 1 ). The average porosity, $\left\langle P_{\mathrm{por}}\right\rangle$, is defined by

$$
<P_{\mathrm{por}}>=\sum_{\mathrm{i}} \frac{t_{\mathrm{i}} \times P_{\mathrm{por}, \mathrm{i}}}{t_{\mathrm{tot}}}
$$

where $t_{\mathrm{i}}$ is thickness of $i$-th MAO layer, and $t_{\mathrm{tot}}$ is total thickness of MAO film. The calculated $\left\langle P_{\text {por }}>\right.$ tends to be larger with increasing the concentration of aluminum nitrate in the precursor solution (Table 2). This tendency indicates that the density of aluminum oxide framework in MAO increases with increasing the aluminum nitrate in the precursor solution. The aluminum oxide framework in A103 and A105 would possess a large
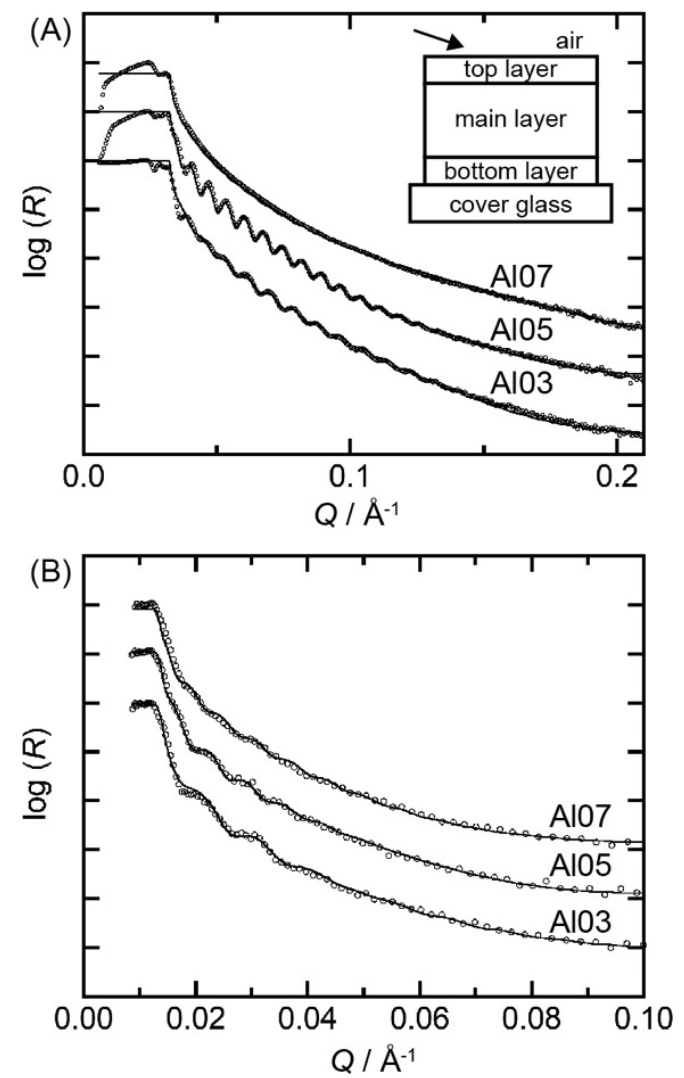

Fig. 3 (A) XRR and (B) NR profiles for Al03, Al05, and Al07. All measurements were performed in air. The solid lines show the fit of the reflectivity data. Inset of (A) shows a model used for the analysis of reflectivity data. The arrow in the inset represents incidences of neutron and X-ray beam.

number of micropores, and the number of micropores would be relatively lower for the framework in A107. This difference in the aluminum oxide framework was also supported by the better structural stability of the MAO film in Al07. When SEM observation was performed after immersion of Al05 and Al07 in an aqueous solution for a few days, A107 exhibited longer time stability of the pore structure.

The $t_{\text {tot }}$ and $\left\langle P_{\text {por }}>\right.$ values derived from XRR and NR are almost the same for A105 and Al07 (Table 2). On the other hand, those for Al03 are somewhat different. We consider that this difference is due to structural nonuniformity of the MAO film for Al03 as shown in the SEM image (Fig. 2(A)).

Our previous study reported that the MAO film in A105 has perpendicularly oriented cylindrical mesopore channels. ${ }^{19}$ This pore structure was confirmed by the absence of Bragg peaks due to out-of-plane mesoscale periodicity in NR and GI-SAXS data. Similarly, the present A105 and Al07 exhibited no Bragg peaks in NR and XRR profiles (Fig. 3). It hence can be considered that the pore structure of the MAO film in Al07 is the same as that in Al05. This pore structure for Al07 is supported by the SEM cross-sectional view (Fig. 2(D)). The pore channels appear to be oriented perpendicular to the substrate surface.

\section{In-situ NR for MAO film on Si disc}

The characterization of MAO film on the glass substrate confirmed that the stable MAO film could be formed by using a precursor solution containing $0.7 \mathrm{M}$ aluminum nitrate. This precursor solution was hence used for deposition of MAO film on a Si disc. The resulting sample (A107/Si) shows clear Kiessig 

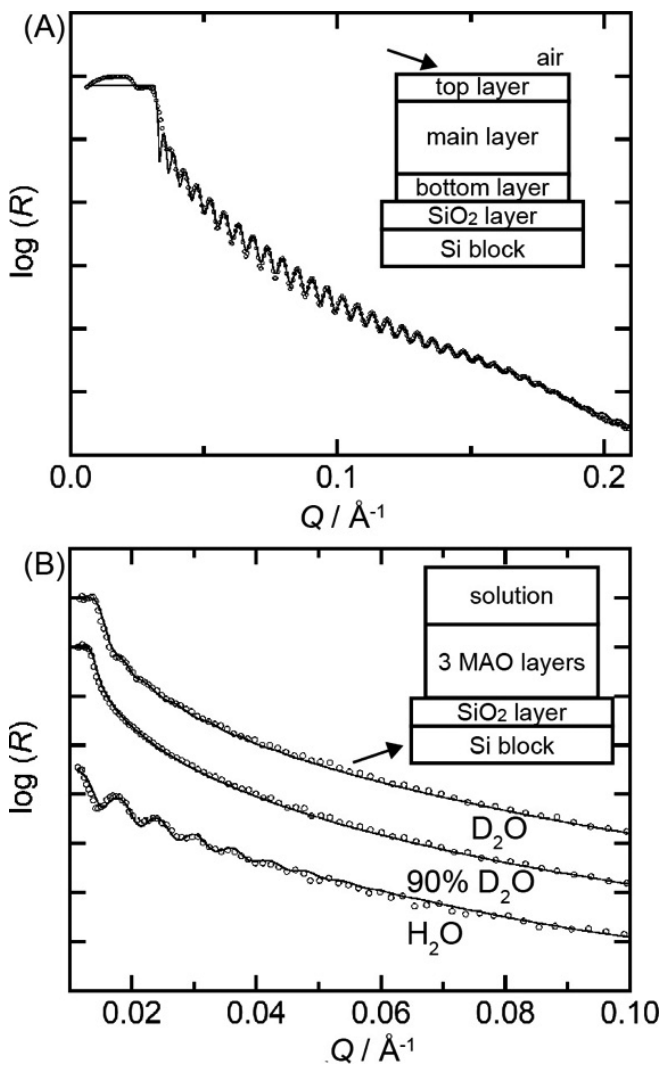

Fig. 4 (A) XRR profile for A107/Si in air, and (B) NR profiles for Al07/Si in $\mathrm{D}_{2} \mathrm{O}, 90 \% \mathrm{D}_{2} \mathrm{O}$, and $\mathrm{H}_{2} \mathrm{O}$. The solid lines show the fit of the reflectivity data. Inset shows a model used for the analysis of XRR and NR data. The arrow in inset represents incidences of neutron and $\mathrm{X}$-ray beam.

fringes in the XRR profile obtained in air (Fig. 4(A)). The XRR for A107/Si shows no Bragg peaks due to out-of-plane mesoscale periodicity. The appearance of Kiessig fringes is due to the SLD contrasts between MAO film and Si disc (Tables 1 and 2). Since thermal oxidation of the silicon surface was expected during the calcination of $\mathrm{Al} 07 / \mathrm{Si}$, we added $\mathrm{SiO}_{2}$ layer on the silicon disc for the analysis of XRR data as schematically shown in the inset of Fig. 4.

After the XRR measurement in air, A107/Si was applied for the in-situ NR experiment. Figure 4(B) shows NR profiles for Al07/Si in $\mathrm{D}_{2} \mathrm{O}, 90 \% \mathrm{D}_{2} \mathrm{O}$, and $\mathrm{H}_{2} \mathrm{O}$. Kiessig fringes shown in $\mathrm{D}_{2} \mathrm{O}$ and $\mathrm{H}_{2} \mathrm{O}$ disappeared for $90 \% \mathrm{D}_{2} \mathrm{O}$. In addition, the NR profile for $\mathrm{Al} 107 / \mathrm{Si}$ in $90 \% \mathrm{D}_{2} \mathrm{O}$ was essentially the same as that for the $\mathrm{Si}$ disc in $90 \% \mathrm{D}_{2} \mathrm{O}$. These results indicate that the contrast of the aluminum oxide matrix in the MAO film is matched to that of $90 \% \mathrm{D}_{2} \mathrm{O}$ (Table 1). The SLD of the aluminum oxide matrix in the MAO film was therefore fixed to be $5.67 \times 10^{-6} \AA^{-2}$ for the analysis of the in-situ NR profiles.

The three NR data sets were simultaneously refined with thickness, volume fraction of solvent in each MAO layer, and surface roughness parameters linked in a single model. In the co-refinement, structural parameters of the $\mathrm{SiO}_{2}$ layer were fixed to those derived from XRR. The structural parameters obtained by the co-refinement are listed in Table 3. Herein, the volume fraction of solvent in the MAO layer is regarded to be equal to porosity. The thicknesses of each MAO layer were almost the same as those derived from XRR, but porosity values were different between NR and XRR. A plausible reason for this discrepancy is the contribution of water molecules to the
Table 3 Structural parameters for Al07/Si obtained from fitting the NR and XRR profiles

\begin{tabular}{llcc}
\hline & & XRR & NR \\
\hline \multirow{2}{*}{ Top } & $t^{\mathrm{a}}$ & $60 \pm 1$ & $60 \pm 3$ \\
& $P_{\text {por }}{ }^{\mathrm{b}}$ & $0.44 \pm 0.01$ & $0.56 \pm 0.01$ \\
& $\sigma^{\mathrm{c}}$ & $6.5 \pm 0.1$ & $2.0 \pm 0.1$ \\
Main & $t$ & $978 \pm 1$ & $989 \pm 5$ \\
& $P_{\text {por }}$ & $0.47 \pm 0.02$ & $0.63 \pm 0.01$ \\
& $\sigma$ & $6.2 \pm 0.1$ & $9.5 \pm 4.3$ \\
Bottom & $t$ & $27 \pm 1$ & $26 \pm 7$ \\
& $P_{\text {por }}$ & $0.31 \pm 0.01$ & $0.55 \pm 0.02$ \\
& $\sigma$ & $25 \pm 1$ & $10 \pm 6$ \\
$\mathrm{SiO}_{2}$ & $t$ & $15 \pm 1$ & 15 \\
& $\sigma$ & $12 \pm 1$ & $1.3 \pm 0.6$ \\
$\mathrm{~T}_{\text {Total }}$ & $t_{\text {tot }}$ & $1065 \pm 3$ & $1075 \pm 9$ \\
& $<P_{\text {por }}>^{\mathrm{d}}$ & 0.46 & 0.62 \\
\hline
\end{tabular}

a. Thickness $(\AA)$.

b. Porosity for XRR was calculated by Eq. (2), and porosity for NR was equal to volume fraction of solvent at each MAO layer. c. Surface roughness $(\AA)$.

d. Average porosity calculated by sum of $\left(t_{\mathrm{i}} \times P_{\mathrm{por}}\right) / t_{\mathrm{tot}}$.

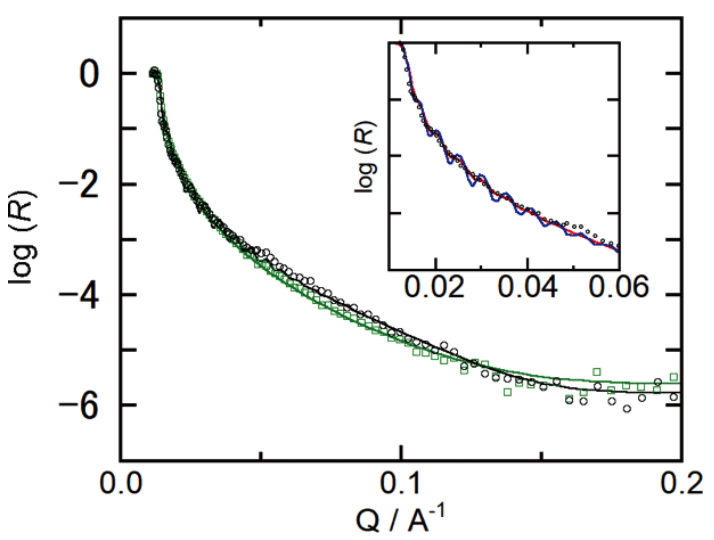

Fig. 5 Comparison of NR profiles obtained in $90 \% \mathrm{D}_{2} \mathrm{O}$ solution (green squares) and in $90 \% \mathrm{D}_{2} \mathrm{O}$ solution containing $10 \mathrm{wt} \%$ GOD (black circles). The green and black solid lines show the fit of the NR data. Inset shows expanded NR profile in $90 \% \mathrm{D}_{2} \mathrm{O}$ solution containing GOD. Blue solid line in inset shows the fit of the NR data by fixing $Q$ resolution derived from instrumental setup (8\%). Red solid line in inset shows the fit by treating $Q$ resolution as variable parameter (14\%).

XRR profile. Since the XRR measurement was performed in air, it can be considered that water molecules adsorb within the hydrophilic pore surfaces of MAO film. The difference of $\mathrm{X}$-ray SLD values between $\mathrm{H}_{2} \mathrm{O}$ and $\mathrm{Al}_{2} \mathrm{O}_{3}$ is not so large; the $\mathrm{X}$-ray SLD values are $9.5 \times 10^{-6} \AA^{-2}$ for $\mathrm{H}_{2} \mathrm{O}$ and $33.3 \times$ $10^{-6} \AA^{-2}$ for $\mathrm{Al}_{2} \mathrm{O}_{3}$, respectively. The water molecules within the pores would increase the SLD of each MAO layer.

After injection of $10 \mathrm{wt} \%$ GOD solution $\left(90 \% \mathrm{D}_{2} \mathrm{O}\right)$ into the measurement cell, the sample cell was stored for $60 \mathrm{~min}$. Then, the NR measurement was performed in the GOD solution. As shown in Fig. 5, a slight difference was recognized at $Q$ around $0.6 \AA^{-1}$ for NR profiles obtained in $90 \% \mathrm{D}_{2} \mathrm{O}$ and in the GOD solution. When we fitted the NR profile in the GOD solution by assuming GOD adsorption at only the external surface of the MAO film, the overall reflectivity profile could be described. However, Kiesssig fringes at $Q$ around $0.2 \AA^{-1}$ in the refined profile were not seen in the experimental profile. On the other hand, when the $Q$ resolution was considered as variable 


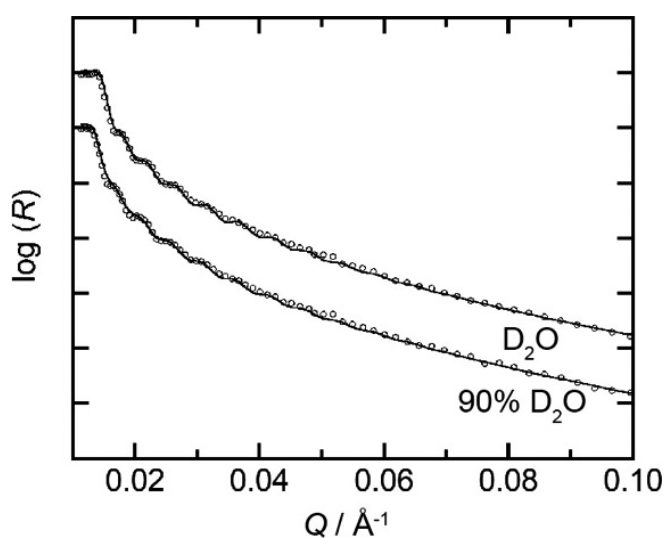

Fig. 6 NR profiles for $\mathrm{A} 107 / \mathrm{Si}$ after rinsing with $\mathrm{D}_{2} \mathrm{O}$. Measurements were performed in $\mathrm{D}_{2} \mathrm{O}$ and $90 \% \mathrm{D}_{2} \mathrm{O}$. The solid lines show the fit of the reflectivity data.

parameter, good agreement between the experimental and refined profiles was obtained with $14 \%$ of $Q$ resolution, which was larger than that derived from the instrumental setup (8\%). These results suggest large inhomogeneities of the GOD layer. Since the NR profile was observed in concentrated GOD solution (10 wt\% GOD solution), stacking or aggregation of GOD molecules at the MAO film surface could be expected. The molecular stacking or aggregation results in large vertical inhomogeniety of the thickness of the GOD layer (surface roughness). In addition, large-scale lateral inhomogeneity of the GOD layer is also expected, because the domain structure of protein molecules at an interface is sometimes more than micrometers. ${ }^{26}$ These inhomogeneities would disturb the resolution of NR, resulting in the disappearance of the Kiessig fringes. ${ }^{27,28}$

The GOD solution in the measurement cell was replaced by the flow of $\mathrm{D}_{2} \mathrm{O}$ solution, and $\mathrm{NR}$ measurement was performed in the $\mathrm{D}_{2} \mathrm{O}$ and $90 \% \mathrm{D}_{2} \mathrm{O}$ solutions (Fig. 6). In the NR profiles after removal of the GOD solution, clear Kiessig fringes were recognized (Fig. 6). The appearance of the Kiessig fringes indicates that the homogeneity of the GOD layer becomes improved during the removal of the GOD solution, that is, excess amount of GOD adsorbed was removed. Co-refinement of both NR data was hence performed by fixing the $Q$-resolution to be $8 \%$. The multilayer model composed of one GOD layer on the MAO film could be used to describe the experimental data (Fig. 6). It can therefore be concluded that majority of the GOD molecules adsorb at the external surface of the MAO film, and penetration of GOD molecules hardly occurs in the concentrated (10 wt $\%$ ) GOD solution.

SLD profiles obtained by the co-refinement of NR data are shown in Fig. 7, together with that obtained for the GOD solution with $14 \%$ of $Q$ resolution. Figure 8 shows the schematic illustrations of the distribution of GOD molecules at the MAO films. Since the SLD of GOD is smaller than those for other matrices (Table 1), the GOD layer is recognized as a dip at the top surface of the MAO film. The dip obtained after the rinsing process is shallower than that in the GOD solution, indicating removal of the excess amount of GOD adsorbed. On the assumption that SLD of GOD was $3.5 \times 10^{-6} \AA^{-2}{ }^{29}$ we calculated the amount of GOD, $A_{\mathrm{GOD}}$, at the MAO surface by using Eq. (3).

$$
A_{\mathrm{GOD}}=\frac{M_{\mathrm{w}}\left(1-f_{\mathrm{sol}}\right) t_{\mathrm{GOD}}}{V_{\mathrm{GOD}} N_{\mathrm{A}}}
$$

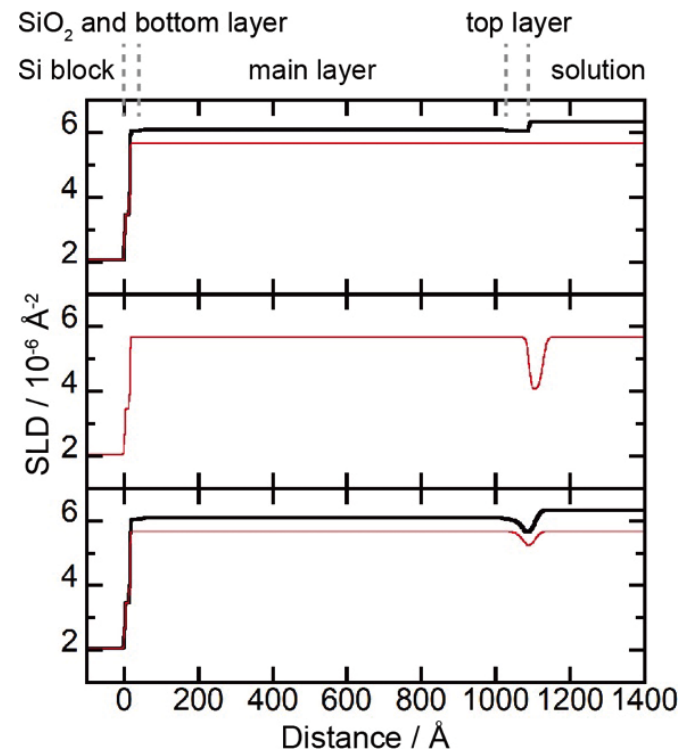

Fig. 7 SLD profiles of the models used for fitting the respective NR data for (top) before GOD adsorption, (middle) for GOD solution, and (bottom) after rinse. The black and red solid lines are respectively for $\mathrm{D}_{2} \mathrm{O}$ and $90 \% \mathrm{D}_{2} \mathrm{O}$.
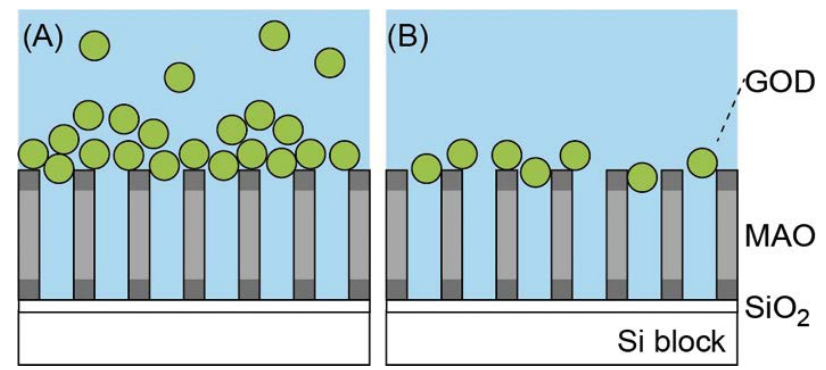

Fig. 8 Schematic illustrations of the distribution of GOD molecules at the MAO film in $10 \mathrm{wt} \%$ GOD solution and in water after removal of excess amount of GOD adsorbed.

Where $M_{\mathrm{w}}, V_{\mathrm{GOD}}$, and $N_{\mathrm{A}}$ are molecular weight of GOD $(160 \mathrm{kDa})$, volume of single GOD molecule $\left(145000 \AA^{3}\right),{ }^{29}$ and Avogadro's number, respectively. $t_{\mathrm{GOD}}$ and $f_{\text {sol }}$ are thickness of GOD layer and volume fraction of solvent in the GOD layer, respectively (Table 3). The amount of GOD at the MAO surface was estimated to be $8.0 \times 10^{-13} \mathrm{~mol} \mathrm{~cm}^{-2}$. By assuming that the area occupied by a single GOD is square of the GOD diameter (65 $\AA),{ }^{29}$ the surface coverage of GOD was calculated to be 0.2 from the amount of GOD at the surface of the MAO film. This coverage value suggests that the GOD molecules located at the MAO film surface without significant aggregation as schematically shown in Fig. 8.

In unbuffered aqueous solution, the surface of the aluminum oxide framework is positively-charged. ${ }^{19}$ It hence can be considered that the negatively-charged GOD ( $\mathrm{pI}=4.2$ ) adsorb at the external surface of the MAO film by strong electrostatic interaction. This strong interaction would prevent the penetration of GOD $\left(60 \times 50 \times 77 \AA^{3}\right)^{22}$ molecules into the aluminum oxide pore channel $(\mathrm{ca} .10 \mathrm{~nm})$. We previously reported that negatively-charged BSA $\left(140 \times 38 \times 38 \AA^{3}\right)^{21}$ could penetrate into an aluminum oxide pore channel with large pore diameter $(33 \mathrm{~nm}) .{ }^{15}$ It hence can be considered that the lack of penetration of GOD for the present MAO film is due to the near matching 
Table 4 Structural parameters for GOD layer onto MAO film

\begin{tabular}{ccc}
\hline & GOD $^{\mathrm{a}}$ & Rinse $^{\mathrm{b}}$ \\
\hline$t^{\mathrm{c}}$ & $38 \pm 2$ & $10 \pm 5$ \\
$f_{\text {sol }}^{\mathrm{d}}$ & $0.34 \pm 0.05$ & $0.31 \pm 1.0$ \\
$\sigma^{\mathrm{e}}$ & $8.2 \pm 3$ & $15 \pm 4$ \\
$\sigma^{\mathrm{f}}$ & $5.2 \pm 4.4$ & $19 \pm 7$ \\
\hline
\end{tabular}

a. In $10 \mathrm{wt} \%$ GOD solution.

b. After rinse by the solution flow.

c. Thickness $(\AA)$.

d. Solvent fraction at GOD layer

e. Roughness at GOD/solvent interface $(\AA)$

f. Roughness at GOD/MAO interface ( $(\AA)$.

of size and curvature between GOD and pore channel. Multiple electrostatic interactions would limit the diffusion of GOD along the narrow pore channel.

In inorganic nanoporous films, penetration of protein molecules into pore channels with diameters of 2.6 to $50 \mathrm{~nm}$ has been studied by utilizing optical interferometry ${ }^{7-9}$ and waveguide mode. ${ }^{15-17}$ These studies reported that the charged protein molecules could penetrate into silica and aluminum oxide pores with opposite surface charges when the pore diameter was larger than the protein dimensions. ${ }^{7,8,15,17}$ No significant penetration of cytochrome $c(12 \mathrm{kDa}, 3.2 \mathrm{~nm}$ in diameter) was reported for mesoporous silica film with $2.6 \mathrm{~nm}$ in pore diameter. ${ }^{9}$ These results imply that the relationship between pore size and protein size is a predominant factor for the penetration of a protein into a small pore. A similar relationship was also predicted for the microscopic study of a single inorganic nanoporous particle. ${ }^{30}$ The present result derived from in-situ NR investigations is the first example that a charged protein cannot penetrate into a relatively large aluminum oxide pore channel with opposite charge. This result suggests that the electrostatic interaction is also a critical factor for the penetration of protein molecules into narrow inorganic pores.

\section{Conclusions}

In the present study, distribution of GOD molecules adsorbed at a MAO film was examined by in-situ NR experiment. The results provided information on the amount and the vertical distribution of GOD molecules adsorbed by the MAO film; there was no evidence of penetration within the nanporous film with the GOD molecules adsorbed exclusively at the external surface of the MAO film. We consider that multiple electrostatic interactions in the narrow pore channel limit the diffusion of GOD along the narrow pore channel.

\section{Acknowledgements}

This work in supported in part by the Japan Society for the Promotion of Science (JSPS) Kakenhi Grant nos. 16H04160 and 17K19022. This work was also partially supported by the Radiation Application Development Association (RADA) under the Support Program for Overseas Facility Users funded by the Ministry of Education, Culture, Sports, Science and Technology of Japan (MEXT). We acknowledge the support of the Australian Centre for Neutron Scattering (beamtime proposal P7962) in providing the neutron research facilities used in this work.

\section{References}

1. J. Nawrocki, J. Chromatogr. A, 1997, 779, 29.

2. K. Ariga, A. Vinu, Y. Yamauchi, Q. Ji, and J. P. Hill, Bull. Chem. Soc. Jpn., 2012, 85, 1.

3. T. Itoh, A. Yamaguchi, T. Kyotani, T. Hanaoka, and F. Mizukami, Adv. Porous Mater., 2016, 4, 157.

4. T. Sato, K. Hata, and K. Nakatani, Anal. Sci., 2017, 33, 647.

5. A. Saini and L. Kisley, J. Appl. Phys., 2019, 126, 081101.

6. W. Ogieglo, H. Wormeester, K.-J. Eichhorn, M. Wessling, and N. E. Benes, Prog. Polym. Sci., 2015, 42, 42.

7. M. Y. Chen and M. J. Sailor, Anal. Chem., 2011, 83, 7186.

8. L. M. Karlsson, P. Tengvall, I. Lundström, and H. Arwin, J. Colloid Interface Sci., 2003, 266, 40.

9. J. Deere, M. Serantoni, K. J. Edler, B. K. Hodnett, J. G. Wall, and E. Magner, Langmuir, 2004, 20, 532.

10. S. A. Bhakta, T. E. Benavidez, and C. D. Garcia, J. Colloid Interface Sci., 2014, 430, 351.

11. P. W. Akers, A. J. Dingley, S. Swift, A. R. J. Nelson, J. Martin, and D. J. McGillivray, J. Phys. Chem. B, 2017, 121, 5908.

12. D. A. Doshi, A. M. Dattelbaum, E. B. Watkins, C. J. Brinker, B. I. Swanson, A. P. Shreve, A. N. Parikh, and J. Majewski, Langmuir, 2005, 21, 2865.

13. S. Isaksson, E. B. Watkins, K. L. Browning, T. K. Lind, M. Cárdenas, K. Hedfalk, F. Höök, and M. Andersson, Nano Lett., 2017, 17, 476.

14. A. Christoulaki, A. Chennevière, E. Dubois, and N. Jouault, Nanoscale, 2019, 11, 2148.

15. K. Hotta, A. Yamaguchi, and N. Teramae, ACS Nano, 2012, 6, 1541 .

16. H. Arafune, A. Yamaguchi, K. Hotta, T. Itoh, and N. Teramae, Anal. Sci., 2013, 29, 187.

17. H. Arafune, K. Hotta, T. Itoh, N. Teramae, and A. Yamaguchi, Anal. Sci., 2017, 33, 473.

18. P. W. Akers, A. J. Dingley, S. Swift, A. R. J. Nelson, J. Martin, and D. J. McGillivray, J. Phys. Chem. B, 2017, 121, 5908.

19. Y. Shibuya, K. Katayama, K. Akutsu-Suyama, and A. Yamaguchi, ACS Omega, 2019, 4, 17890.

20. H. Masuda, H. Yamada, M. Satoh, H. Asoh, M. Nakao, and T. Tamamura, Appl. Phys. Lett., 1997, 71, 2770.

21. H. J. Hecht, H. M. Kalisz, J. Hendle, R. D. Schmid, and D. Schomburg, J. Mol. Biol., 1993, 229, 153.

22. P. G. Squire, P. Moser, and C. T. O'Konski, Biochemistry, 1968, 7, 4261.

23. M. James, A. Nelson, S. A. Holt, T. Saerbeck, W. A. Hamilton, and F. Klose, Nucl. Instr. Methods Phys. Res. A, 2011, 632, 112.

24. A. Nelson, J. Appl. Crystallogr., 2006, 39, 273.

25. S. Dourdain, A. Mehdi, J. F. Bardeau, and A. Gibaud, Thin Solid Films, 2006, 495, 205.

26. W. Daear, M. Mahadeo, and E. J. Prenner, Biochim. Biophys. Acta, 2017, 1859, 1749.

27. S. K. Sinha, Y. P. Feng, C. A. Melendres, D. D. Lee, T. P. Russell, S. K. Satija, E. B. Sirota, and M. K. Sanyal, Physica A, 1996, 231, 99.

28. V. P. Romanov, S. V. Ulyanov, V. M. Uzdin, G. Nowak, M. Vadala, and H. Zabel, J. Phys. D, 2008, 41, 115401.

29. J. R. Lu, T.-J. Su, D. Georganopoulou, and D. E. Williams, J. Phys. Chem. B, 2003, 107, 3954.

30. A. M. Clemments, P. Botella, and C. C. Landry, J. Am. Chem. Soc., 2017, 139, 3978. 